\title{
THE SECURITY PROBLEM AGAINST INFERENCE ATTACKS ON OBJECT-ORIENTED DATABASES
}

\author{
Yasunori Ishihara, Toshiyuki Morita and Minoru Ito
}

\begin{abstract}
Inference attacks mean that a user infers (or tries to infer) the result of an unauthorized method execution using only authorized methods to the user. We say that a method $m$ is secure against inference attacks by a user $u$ if there exists no database instance for which $u$ can infer the result of $m$. It is important for database administrators to know which methods are secure and which ones are not. When an administrator finds that a method which retrieves top secret information is not secure against inference attacks by $u$, then the administrator can prevent $u$ from attacking the method by changing the authorization for $u$. This paper formalizes the security problem (i.e., to determine whether given method is secure or not) for method schemas, and presents the following results. First, it is shown that the security problem is undecidable. Next, a decidable sufficient condition for a given method to be secure is proposed. Furthermore, it is shown that the sufficient condition is also a necessary one if a given schema is monadic (i.e., every method has exactly one parameter). The time complexity to decide the condition is also evaluated. For a monadic schema, the condition is decidable (and therefore, the security problem is solvable) in polynomial time of the size of the schema.
\end{abstract}

Keywords: Inference, object-oriented database

\section{INTRODUCTION}

In recent years, various authorization models for object-oriented databases (OODBs) have been proposed and studied. Among of them, the method-based authorization model [4] is one of the most elegant models since it is in harmony with the concept that "an object can be accessed only via its methods" in the object-oriented paradigm. In the model, an authorization $A$ for a user $u$ can be represented as a set of expressions $m\left(c_{1}, \ldots, c_{n}\right)$, which means that $u$ can 


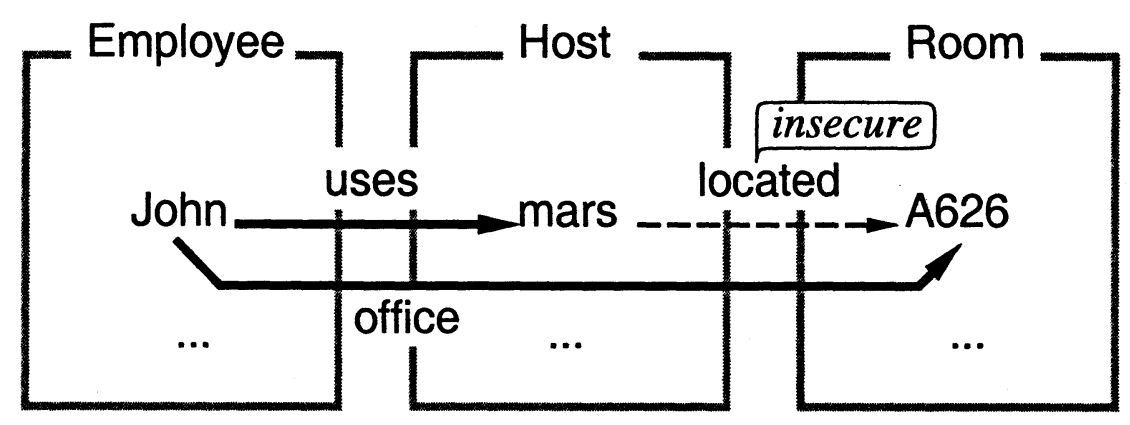

Figure 20.1 An example of an insecure method.

directly invoke method $m$ on any tuple $\left(o_{1}, \ldots, o_{n}\right)$ of objects such that $o_{i}$ is an object of class $c_{i}(1 \leq i \leq n)$. On the other hand, even if $m\left(c_{1}, \ldots, c_{n}\right) \notin A, u$ can invoke $m$ indirectly through another method execution in several models (e.g., protection mode in [3]). Although such indirect invocations are useful for data hiding [3], they may also allow inference attacks in some situations.

Example 7 Let Employee, Host, and Room be classes representing employees, hosts, and rooms, respectively. Suppose that a method uses returns the host which a given employee uses, and a method located returns the room in which a given host is placed. Also suppose that a method office, which returns the room occupied by a given employee, is implemented as office $(x)=\operatorname{located}($ uses $(x)$ ).

Now suppose that the physical computer network is top secret information. In this case, an authorization for a user $u$ may be the one shown in Figure 20.1, where a solid (resp. dotted) arrow denotes an authorized (resp. unauthorized) method to $u$. Suppose that $u$ have obtained that uses(John) = mars and office (John) $=\mathrm{A} 626$ using the authorized methods. Also suppose that $u$ knows the implementation body of office as its behavioral specification. Then, $u$ can infer that located(mars) $=$ A626.

On the other hand, suppose that method uses retrieves top secret information and therefore the authorization of $u$ is set as shown in Figure 20.2. Then, $u$ knows that located(mars) $=\mathrm{A} 626$, office $(J o h n)=\mathrm{A} 626$, and office $(x)=$ located(uses $(x))$, similarly to the former case. However, u cannot conclude that uses(John) = mars only from the above information, since there may be another host $h$ such that uses(John) $=h$ and located $(h)=\mathrm{A} 626$.

For a given database schema $S$ and an authorization $A$ for a user $u$, an $n$-ary method $m$ is said to be secure at $\left(c_{1}, \ldots, c_{n}\right)$ (each $c_{i}$ is a class in $S$ ) against inference attacks by $u$ if $u$ cannot infer the result of $m\left(o_{1}, \ldots, o_{n}\right)$ for any objects $o_{i}$ of class $c_{i}$ in any database instance $I$ of $S$, using only autho- 


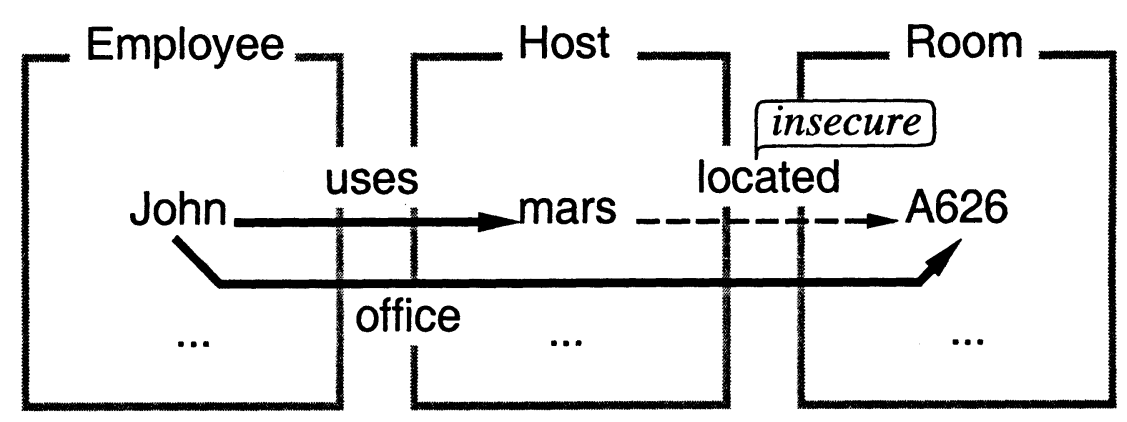

Figure 20.2 An example of a secure method.

rized methods to $u$. Otherwise, $m$ is insecure. For example, if uses(Employee) and office(Employee) are authorized, then located is insecure since the user can infer located(mars) $=$ A626 under the database instance shown in Figure 20.1. On the other hand, it will be shown later that uses is secure when only located(Host) and office(Employee) are authorized. It is important for database administrators to know which methods are secure and which ones are not. When an administrator finds that a method which retrieves top secret information is insecure against inference attacks by $u$, the administrator can prevent $u$ from attacking the method by changing the authorization for $u$.

In this paper, we formally define the security problem, i.e., to determine whether a given method is secure or not. We adopt method schemas proposed by [2] as a formal model of OODB schemas since they support such basic features of OODBs as method overloading, dynamic binding, and complex objects. The semantics is simply defined based on term rewriting. Under this formalization, we first show that the problem is undecidable. Next, we propose a decidable sufficient condition for a method to be secure. Furthermore, we show that the sufficient condition is also a necessary one if a given schema is monadic (i.e., every method has exactly one parameter). Finally, we evaluate the time complexity of deciding the condition. For a monadic method schema, the proposed condition is decidable (and therefore, the security problem is solvable) in polynomial time of the size of the schema.

In this paper we discuss precise inference in OODBs. Precise inference means that a user can infer (or, is interested in) only the exact value of the result of an unauthorized method. On the other hand, most of the recent researches concentrate on imprecise inference in relational databases, not OODBs. Imprecise inference means that a user can infer (or, is interested in) possible values of the result of an unauthorized method (query) with a certain probability. In [5], FD-based imprecise inference involving abduction and partial deduction is discussed. In [12], a quantitative measure of inference risk is formally defined. 
Imprecise inference with external, common sense knowledge can be regarded as data mining $[6,9]$.

[11] focuses on both precise and imprecise inference in OODBs. Besides inferability of the result of a method execution, the article introduces the notion of controllability, which means that a user can control (alter arbitrarily) an attribute-value of an object in a database instance. We do not consider controllability since our query language does not support update operations for database instances. However, since our query language supports recursion while the one in [11] does not, detecting inferability in our formalization is not trivial. [11] also proposes, for a given database schema $S$ and an authorization $A$, a sound algorithm for detecting inferability or controllability. However, [11] does not evaluate the complexity of the algorithm.

\section{METHOD SCHEMAS}

\section{$2.1 \quad$ SYNTAX}

We introduce some notations before defining the syntax of method schemas. Let $F$ be a family of disjoint sets $F_{0}, F_{1}, F_{2}, \ldots$, where $F_{n}(n=0,1,2, \ldots)$ is a set of function symbols of arity $n$. For a countable set $X$ of variables, let $T_{F}(X)$ denote the set of all the terms freely generated by $F$ and $X$. For a set $V$, let $V^{n}$ denote the Cartesian product $\underbrace{V \times \cdots \times V}_{n}$. For a term $t \in T_{F}(X)$, an $n$-tuple $\mathbf{t}=\left(t_{1}, \ldots, t_{n}\right) \in\left(T_{F}(X)\right)^{n}$ of terms, and an $n$ tuple $\mathbf{x}=\left(x_{1}, \ldots, x_{n}\right) \in X^{n}$ of variables, let $t[\mathbf{t} / \mathbf{x}]$ denote the term obtained by simultaneously replacing every $x_{i}$ in $t$ with $t_{i}(1 \leq i \leq n)$. For example, $f\left(x_{1}, g\left(x_{1}, x_{2}\right)\right)\left[\left(f(a), x_{1}\right) /\left(x_{1}, x_{2}\right)\right]=f\left(f(a), g\left(f(a), x_{1}\right)\right)$. For a term $t$, define the set of occurrences $R(t)$ as the smallest set of sequences of positive integers with the following two properties:

- $\varepsilon \in R(t)$, where $\varepsilon$ is the empty sequence.

- If $r \in R\left(t_{i}\right)$, then $i \cdot r \in R\left(f\left(t_{1}, \ldots, t_{n}\right)\right)(1 \leq i \leq n)$, where the center dot "." represents the concatenation of sequences.

An occurrence of $t$ specifies (the position of) a subterm of $t$. For example, $1 \cdot 2$ of $f(f(x, g(x)), g(x)))$ specifies the first $g(x)$. The replacement in $t$ of $t^{\prime}$ at $r$, denoted $t\left[r \leftarrow t^{\prime}\right]$, is defined as follows:

- $t\left[\varepsilon \leftarrow t^{\prime}\right]=t^{\prime}$;

- $f\left(t_{1}, \ldots, t_{i}, \ldots, t_{n}\right)\left[i \cdot r \leftarrow t^{\prime}\right]=f\left(t_{1}, \ldots, t_{i-1}, t_{i}\left[r \leftarrow t^{\prime}\right], t_{i+1}, \ldots, t_{n}\right)$, where $1 \leq i \leq n$.

For example, $f(f(x, g(x)), g(x))[1 \cdot 2 \leftarrow h(a, b)]=f(f(x, h(a, b)), g(x))$. 
We go on to the definition of method schemas. Let $C$ be a finite set of class names (or simply classes) and $M$ a family of mutually disjoint finite sets $M_{0}$, $M_{1}, M_{2}, \ldots$, where $M_{n}(n=0,1,2, \ldots)$ is a set of method names of arity $n$. Each $M_{n}$ is partitioned into $M_{\mathrm{b}, n}$ and $M_{\mathrm{c}, n}$ : Each $m_{\mathrm{b}} \in M_{\mathrm{b}}\left(=\bigcup_{n>0} M_{\mathrm{b}, n}\right)$ (resp. $m_{\mathrm{c}} \in M_{\mathrm{c}}\left(=\bigcup_{n \geq 0} M_{\mathrm{c}, n}\right)$ ) is called a base method name (resp. composite method name). Furthermore, each $m \in M\left(=M_{\mathrm{b}} \cup M_{\mathrm{c}}\right)$ is simply called a method name. We say that $M$ is a method signature.

Hereafter, we often use a bold letter $\mathbf{v}$ to mean $\left(v_{1}, \ldots, v_{n}\right)$ without explicitly defining it when $n$ is irrelevant or obvious from the context.

Definition 15 Let $\mathbf{c} \in C^{n}$. A base method definition of $m_{\mathrm{b}} \in M_{\mathrm{b}, n}$ at $\mathbf{c}$ is a pair $\left(m_{\mathrm{b}}(\mathbf{c}), c\right)$, where $c \in C$. A composite method definition of $m_{\mathrm{c}} \in M_{\mathrm{c}, n}$ at c is a pair $\left(m_{\mathrm{c}}(\mathbf{c}), t\right)$, where $t \in T_{M}\left(\left\{x_{1}, \ldots, x_{n}\right\}\right)$.

Let $o_{i}$ be an object of class $c_{i}(1 \leq i \leq n)$ (see Defs. 16 and 18 for formal definitions). Informally, the above base method definition declares that the application of $m_{\mathrm{b}}$ to $\mathbf{0}=\left(o_{1}, \ldots, o_{n}\right)$ results in an object of $c$ or its subclass, while the above composite method definition states that the application of $m_{\mathrm{c}}$ to $\mathbf{o}$ results in term rewriting starting from $t[\mathbf{o} / \mathbf{x}]$. The formal definition is presented in Section 2.2.

Definition $16 A$ method schema $[1,2] S$ is a 5-tuple $\left(C, \leq, M, \Sigma_{\mathrm{b}}, \Sigma_{\mathrm{c}}\right)$, where:

1. $C$ is a finite set of class names.

2 . $\leq$ is a partial order representing a class hierarchy. When $c^{\prime} \leq c$, we say that $c^{\prime}$ is a subclass of $c$ and $c$ is a superclass of $c^{\prime}$. We naturally extend $\leq$ to $n$-tuples of classes as follows: For two tuples $\mathbf{c}=\left(c_{1}, \ldots, c_{n}\right)$ and $\overline{\mathbf{c}^{\prime}}=\left(c_{1}^{\prime}, \ldots, c_{n}^{\prime}\right)$, we write $\mathbf{c} \leq \mathbf{c}^{\prime}$ iff $c_{i} \leq c_{i}^{\prime}$ for all $i$.

3. $M$ is a method signature.

4. $\Sigma_{\mathrm{b}}$ is a set of base method definitions.

5. $\Sigma_{\mathrm{c}}$ is a set of composite method definitions.

For each possible combination $\mathbf{c} \in C^{n}$ and $m \in M_{n}$, there must exist at most one method definition of $m$ at $\mathbf{c}$. If every method of $S$ has exactly one parameter, then $S$ is monadic.

\subsection{SEMANTICS}

Method definitions are inherited along the class hierarchy. 
Definition 17 Let $S=\left(C, \leq, M, \Sigma_{\mathrm{b}}, \Sigma_{\mathrm{c}}\right), m_{\mathrm{b}} \in M_{\mathrm{b}, n}$, and $\mathbf{c} \in C^{n}$. Suppose that $\left(m_{\mathrm{b}}\left(\mathbf{c}^{\prime}\right), c^{\prime}\right) \in \Sigma_{\mathrm{b}}$ is the base method definition of $m_{\mathrm{b}}$ at the smallest $\mathbf{c}^{\prime}$ above $\mathbf{c}$, i.e., whenever $\left(m_{\mathrm{b}}\left(\mathbf{c}^{\prime \prime}\right), c^{\prime \prime}\right) \in \Sigma_{\mathrm{b}}$ and $\mathbf{c} \leq \mathbf{c}^{\prime \prime}$, it is the case that $\mathbf{c}^{\prime} \leq \mathbf{c}^{\prime \prime}$. We define the resolution $\operatorname{Res}\left(m_{\mathrm{b}}(\mathbf{c})\right)$ of $m_{\mathrm{b}}$ at $\mathbf{c}$ as $c^{\prime}$. If such a unique base method definition does not exist, then $\operatorname{Res}\left(m_{\mathrm{b}}(\mathbf{c})\right)$ is undefined, denoted $\perp$. The resolution of a composite method is defined in the same way.

The semantics of a method schema is defined as follows. To each class name, a set of objects is assigned. Also, to each base method name $m_{\mathrm{b}}$, a mapping over appropriate sets of objects is assigned as its interpretation. The semantics of a composite method is defined by the interpretation of base methods and term rewriting.

Definition 18 An interpretation (or, also called a database instance) of a method schema $S$ is a pair $I=(\nu, \mu)$ with the following properties:

1. To each $c \in C, \nu$ assigns a finite disjoint set $\nu(c)$ of object identifiers (or simply, objects). Each $o \in \nu(c)$ is called an object of class $c$. Let $O_{I}=$ $\bigcup_{c \in C} \nu(c)$. For $\mathbf{c}=\left(c_{1}, \ldots, c_{n}\right)$, let $\nu(\mathbf{c})$ denote $\nu\left(c_{1}\right) \times \cdots \times \nu\left(c_{n}\right)$.

2. For each $m_{\mathrm{b}} \in M_{\mathrm{b}, n}, \mu\left(m_{\mathrm{b}}\right)$ is a partial mapping from $O_{I}^{n}$ to $O_{I}$ which satisfies the following two conditions. Let $\mathbf{c}, \mathbf{c}^{\prime} \in C^{n}$.

(a) If $\operatorname{Res}\left(m_{\mathrm{b}}(\mathbf{c})\right)=c^{\prime}$, then $\left.\mu\left(m_{\mathrm{b}}\right)\right|_{\nu(\mathbf{c})}$ is a total mapping to $\bigcup_{c \leq c^{\prime}} \nu(c)$, where "|" denotes that the domain of $\mu$ is restricted to $\nu(\mathbf{c})$.

(b) If $\operatorname{Res}\left(m_{\mathrm{b}}(\mathbf{c})\right)=\perp$, then $\mu\left(m_{\mathrm{b}}\right)$ is undefined everywhere in $\nu(\mathbf{c})$.

A term in $T_{M}\left(O_{I}\right)$ is called an instantiated term. That is, an instantiated term consists of method names in $M$ and objects in $O_{I}$. The one-step execution relation $\rightarrow_{I}$ on the instantiated terms, based on the leftmost innermost reduction strategy, is defined as follows:

Definition 19 Let $m(\mathbf{0})(\mathbf{o} \in \nu(\mathbf{c}))$ be the subterm of $t \in T_{M}\left(O_{I}\right)$ at the leftmost innermost occurrence $r$.

1. If $m \in M_{\mathrm{b}}$ and $\operatorname{Res}(m(\mathbf{c})) \neq \perp$, then $t \rightarrow_{I} t[r \leftarrow \mu(m)(\mathbf{o})]$.

2. If $m \in M_{\mathrm{c}}$ and $\operatorname{Res}(m(\mathbf{c}))=t^{\prime} \neq \perp$, then $t \rightarrow_{I} t\left[r \leftarrow t^{\prime}[\mathbf{o} / \mathbf{x}]\right]$.

Note that, by Def. 19, for any instantiated term $t$, there exists at most one term $t^{\prime}$ such that $t \rightarrow_{I} t^{\prime}$. That is, every execution is deterministic.

Let $\rightarrow_{I}^{*}$ be the reflexive and transitive closure of $\rightarrow_{I}$. If $t \rightarrow_{I}^{*} t^{\prime}$ and there exists no $t^{\prime \prime}$ such that $t^{\prime} \rightarrow_{I} t^{\prime \prime}$, then $t^{\prime}$ is called the execution result of $t$, and we 
write $t \downarrow=t^{\prime}$. If $t \downarrow \in O_{I}$, then the execution of $t$ is successful, and if $t \downarrow \notin O_{I}$ because of nonexistence of the resolution, then the execution of $t$ is aborted. In both cases (i.e., if $t \downarrow$ exists), the execution of $t$ is terminating. On the other hand, if $t \downarrow$ does not exist, then the execution of $t$ is nonterminating. We omit the subscript $I$ and simply write $\rightarrow$ or $\rightarrow^{*}$ if $I$ is understood from the context.

\section{INFERENCE ATTACKS}

\subsection{AUTHORIZATION}

Various sophisticated method-based authorization models for OODBs have been proposed. In this paper, however, discussing authorization models is not our main purpose, and therefore we adopt the following simple but general authorization model.

Definition 20 Let $S=\left(C, \leq, M, \Sigma_{\mathrm{b}}, \Sigma_{\mathrm{c}}\right)$. An authorization $A$ for a user $u$ under $S$ is a finite set of $m(\mathbf{c})$, where $m \in M_{n}$ and $\mathbf{c} \in C^{n}$. Intuitively, $m(\mathbf{c}) \in$ $A$ means that $u$ is authorized to directly invoke method $m$ on any tuple $\mathbf{0}$ of objects such that $\mathbf{0} \in \nu(\mathbf{c})$.

An authorization is often modeled as a pair of a base authorization and a set of inference rules. An example of an inference rule is "if $u$ is authorized to invoke $m$ on objects of $c$, then $u$ is also authorized to invoke $m$ on objects of the subclasses of $c$." When $c_{1} \leq c$ and $c_{2} \leq c$, the base authorization $\{m(c)\}$ is expanded into $\left\{m(c), m\left(c_{1}\right), m\left(c_{2}\right)\right\}$ by this rule. In this paper, we assume that a given authorization has already been expanded.

\subsection{FORMAL DEFINITION OF USER'S INFERENCE}

In this paper, information provided by a database is modeled as a set of (in)equalities. For example, suppose that a user $u$ executes office(John) and obtains the result A626. In this case, the information that $u$ obtains is office(John) $\downarrow=A 626$. In what follows, we demonstrate that user's inference can be formalized as the congruence closure of a finite set of ground equalities when the two reasonable conditions $(\mathrm{Q} 1)$ and $(\mathrm{Q} 2)$ stated below are satisfied.

First of all, we define the information which $u$ can obtain directly from a database instance $I=(\nu, \mu)$.

(*1) User $u$ obtains $m(\mathbf{o}) \downarrow=o$ iff it is the case that $m(\mathbf{c}) \in A, \mathbf{o} \in \nu(\mathbf{c})$, and $m(\mathbf{o}) \downarrow=o \in O_{I}$. That is, $u$ knows what the result of $m(\mathbf{0})$ is if executing $m(\mathbf{o})$ is authorized and the execution is successful.

(*2) User $u$ obtains $\operatorname{Res}(m(\mathbf{c}))=t$ iff it is the case that $m(\mathbf{c}) \in A$ and $\operatorname{Res}(m(\mathbf{c}))=t$. That is, $u$ knows the type declaration of $m$ at $\mathbf{c}$ (when $m$ 
is a base method) or the behavioral specification of $m$ at $\mathbf{c}$ (when $m$ is a composite method), if executing $m(\mathbf{o})(\mathbf{o} \in \nu(\mathbf{c}))$ is authorized.

In Example 7, (*1) and (*2) are stated informally.

Next, suppose that $u$ can use at least four inference rules: reflexivity, symmetry, transitivity, and substitutivity (i.e., if $t_{i}=t_{i}^{\prime}$ for all $i$, then $f(\mathbf{t})=f\left(\mathbf{t}^{\prime}\right)$ ). Also suppose that user $u$ knows that $o \neq o^{\prime}$ for distinct objects $o$ and $o^{\prime}$ (e.g., $u$ knows John $\neq$ Alice, Sara $\neq$ A626, and so on).

The goal of inference attacks is to obtain $o$ such that $m(\mathbf{o}) \downarrow=o$ for some $m$ and $\mathbf{o}$. In other words, $u$ wants to infer equalities. Therefore, if we find a reasonable condition under which inequalities are useless to infer equalities, we can formalize user's inference as the congruence closure of equalities induced by $(* 1)$ and $(* 2)$. Let us examine the following example:

Example 8 Recall the second case of Example 7, where $u$ cannot infer uses(John) $\downarrow=$ mars since there may be another host $h$ such that uses(John) $\downarrow=h$ and located $(h) \downarrow=\mathrm{A} 626$. However, if $u$ knows that located $(o) \downarrow \neq A 626$ for any other object $o$ in the database instance, then $u$ can conclude that uses $($ John $)=$ mars.

The above example suggests that inequalities are useless to infer equalities if the following condition is satisfied:

(Q1) User $u$ does not know what $O_{I}$ is.

In many cases, this condition is satisfied by just hiding $O_{I}$ from the user.

Equalities obtained by $(* 2)$ are not ground (i.e., include variables). However, together with the following condition (Q2), they are equivalent to a finite set of ground equalities, which has many good properties:

(Q2) The user does not know what $C$ is.

This condition is also satisfied by just hiding $C$.

Example 9 Consider a schema with a composite method $m_{\mathrm{c}}$ which has the same resolution t at every class $c \in C$. Let $A=\left\{m_{\mathrm{c}}(c) \mid c \in C\right\}$ be an authorization for a user $u$.

Assume that $u$ knows what $C$ is. Then, $u$ can infer that $m_{\mathrm{c}}\left(t^{\prime}\right) \downarrow=t\left[t^{\prime} / x\right] \downarrow$ for any term $t^{\prime}$ such that $t^{\prime} \downarrow \in O_{I}$, since $m_{\mathrm{c}}$ has the same resolution $t$ at any class. Note that, in this inference, $u$ does not need to know which class $t^{\prime} \downarrow$ belongs to.

On the other hand, if $(Q 2)$ is satisfied, then $u$ cannot conclude that $m_{\mathrm{c}}\left(t^{\prime}\right) \downarrow=t\left[t^{\prime} / x\right] \downarrow$ without exactly inferring the class to which $t^{\prime} \downarrow$ belongs 
since there may be another class $c$ in $C$ such that $t^{\prime} \downarrow \in \nu(c)$ and $\operatorname{Res}\left(m_{\mathrm{c}}(c)\right) \neq$ $t$.

Type checking $[2,10]$ is useless when $(\mathrm{Q} 2)$ is satisfied. Therefore, to know the class to which $t^{\prime} \downarrow$ belongs is to infer the exact value of $t^{\prime} \downarrow$. Thus, the equalities obtained by $(* 2)$ can be applied only to terms $t^{\prime}$ such that $t^{\prime} \downarrow$ is known. This means that each equality $\operatorname{Res}\left(m_{\mathrm{c}}(\mathbf{c})\right)=t$ obtained by $(* 2)$ can be regarded as $\left\{m_{\mathrm{c}}(\mathbf{o}) \downarrow=t[\mathbf{o} / \mathbf{x}] \downarrow \mid \mathbf{o} \in \nu(\mathbf{c})\right\}$, which is a finite set of ground equalities.

Consequently, by assuming $(\mathrm{Q} 1)$ and $(\mathrm{Q} 2)$, we can model user's inference as the congruence closure of a finite set of ground equalities induced by $(* 1)$ and $(* 2)$. For technical reasons, we define the congruence closure through rewriting rules $\triangleright_{I, A}$ introduced below. From the correctness of Knuth-Bendix completion [8], $t \downarrow=o$ iff $t$ is reducible to $o$ by $\triangleright_{I, A}$.

Definition 21 Define $P_{I, A}$ as the minimum set of rewriting rules $\triangleright_{I, A}$ on $T_{M}\left(O_{I}\right)$ satisfying the following three conditions. Intuitively, $t \triangleright_{I, A} O$ means that the user knows or can infer that $t \downarrow=o$.

(A) If $m(\mathbf{c}) \in A, \mathbf{o} \in \nu(\mathbf{c})$, and $m(\mathbf{o}) \downarrow=o \in O_{I}$, then $P_{I, A}$ contains

$$
m(\mathbf{o}) \triangleright_{I, A} \text { o. }
$$

This corresponds to $(* 1)$.

(B) If $m_{\mathrm{c}}(\mathbf{c}) \in A, m_{\mathrm{c}} \in M_{\mathrm{c}}, \mathbf{o} \in \nu(\mathbf{c}), m_{\mathrm{c}}(\mathbf{o}) \downarrow=o \in O_{I}$, and $\operatorname{Res}\left(m_{\mathrm{c}}(\mathbf{c})\right)=$ $t \neq \perp$, then $P_{I, A}$ contains

$$
t[\mathbf{o} / \mathbf{x}] \triangleright_{I, A} o .
$$

This essentially corresponds to $(* 2)$.

(C) If $P_{I, A}$ contains $t \triangleright_{I, A}$ o and $t^{\prime \prime} \triangleright_{I, A} o^{\prime \prime}$ such that $t^{\prime \prime}$ is a proper subterm of $t$ at $r^{\prime \prime}$, then $P_{I, A}$ contains

$$
t\left[r^{\prime \prime} \leftarrow o^{\prime \prime}\right] \triangleright_{I, A} o .
$$

This simulates Knuth-Bendix completion procedure.

By definition, the right-hand side of each rule is an object. Note that the existence of $t \triangleright_{I, A} o$ in $P_{I, A}$ implies $t \rightarrow_{I}^{*} o$.

Define $\Rightarrow_{I, A}$ as the one-step reduction relation by $\triangleright_{I, A}$. That is, $t \Rightarrow_{I, A} t^{\prime}$ iff there exists a subterm $t^{\prime \prime}$ of $t$ at $r^{\prime \prime}$ such that $t^{\prime \prime} \triangleright_{I, A} o^{\prime \prime} \in P_{I, A}$ and $t^{\prime}=$ $t\left[r^{\prime \prime} \leftarrow o^{\prime \prime}\right]$. Let $\Rightarrow_{I, A}^{*}$ denote the reflexive and transitive closure of $\Rightarrow_{I, A}$. For readability, we often write $\triangleright_{I}$ and $P_{I}$ instead of $\triangleright_{I, A}$ and $P_{I, A}$, respectively. 


\subsection{THE SECURITY PROBLEM}

The notion of security of methods discussed in Introduction is naturally extended to terms in $T_{M}(C)$ as follows: A term $\tau \in T_{M}(C)$ is said to be secure if there exists no interpretation $I=(\nu, \mu)$ such that $\tau[\mathbf{o} / \mathbf{c}] \Rightarrow_{I, A}^{*} o$ for any $\mathbf{o} \in \nu(\mathbf{c})$ and $o \in O_{I}$. Otherwise, $\tau$ is insecure. The security problem is to determine whether a given $\tau \in T_{M}(C)$ is secure or not.

Theorem 1 The security problem for method schemas with methods of arity two is undecidable.

Sketch of Proof: The type-consistency problem is to determine whether, for a given method schema $S$, there exists an interpretation of $S$ which causes an aborted execution. [7] shows that the problem for method schemas with methods of arity two is undecidable by reducing the Post's Correspondence Problem (PCP) to the problem. In the reduction, each interpretation $I$ is regarded as a candidate for a solution to a PCP. If $I$ is actually a solution, then execution of a term, say $m(o)$, is aborted under $I$. Otherwise, $m(o)$ is nonterminating. By slightly modifying the reduction in [7], we can construct a schema with the following properties:

- If $I$ is a solution, then the execution of a term, say $m^{\prime}(o)$, is successful under $I$.

- Otherwise, $m^{\prime}(o)$ is nonterminating under $I$.

Let $c$ be the class to which $o$ belongs. Let $A=\left\{m^{\prime}(c)\right\}$ and $\tau=m^{\prime}(c)$. Then, the PCP has a solution iff there exists $I=(\nu, \mu)$ such that $\tau[o / c] \Rightarrow_{I}^{*} o^{\prime}$ for some $o$ and $o^{\prime}$.

\section{SECURITY ANALYSIS}

\subsection{A SUFFICIENT CONDITION}

In this section we propose a decidable sufficient condition for a given term $\tau \in T_{M}(C)$ to be secure. The main idea is to introduce new rewriting rules on $T_{M}(C)$ which "conservatively" approximate $\triangleright_{I, A}$, i.e., if $\tau$ is insecure, then $\tau$ is reducible to a class $c$ by the new rewriting rules. Intuitively, each $t \in T_{M}(C)$ is considered as the set of instantiated terms $\{t[\mathbf{o} / \mathbf{c}] \mid \mathbf{o} \in \nu(\mathbf{c})\}$. The "execution result" $E(t)$ of $t$ is defined as follows: $c \in E(t)$ iff there exists an interpretation $I=(\nu, \mu)$ such that $t[\mathbf{o} / \mathbf{c}] \downarrow \in \nu(c)$ for some $\mathbf{o} \in \nu(\mathbf{c})$. Unfortunately, we cannot compute $E$ exactly in general [2]. However, we can compute $Z: T_{M}(C) \rightarrow 2^{C}$ such that $Z(t) \supseteq E(t)$ for every $t \in T_{M}(C)$ [10]. We use such $Z$ to approximate $\triangleright_{I, A}$. The smaller $Z(t)$ is, the better approximation we 
have, although the approximation is still conservative even when $Z(t)=C$ for all $t$. The algorithm in [10] gives a fairly small $Z$.

The next definition introduces the new rewriting rules $\triangleright_{S, A, Z}$ on $T_{M}(C)$ which approximate $\triangleright_{I, A}$.

Definition 22 Define $P_{S, A, Z}$ as the minimum set of rewriting rules $\triangleright_{S, A, Z}$ on $T_{M}(C)$ satisfying the following three conditions:

(A) If $m(\mathbf{c}) \in A$, then $P_{S, A, Z}$ contains

$$
m(\mathbf{c}) \triangleright_{S, A, Z} c
$$

for each $c \in Z(m(\mathbf{c}))$.

(B) If $m_{\mathrm{c}}(\mathbf{c}) \in A, m_{\mathrm{c}} \in M_{\mathrm{c}, n}$, and $\operatorname{Res}\left(m_{\mathrm{c}}(\mathbf{c})\right)=t \neq \perp$, then $P_{S, A, Z}$ contains

$$
t[\mathbf{c} / \mathbf{x}] \triangleright_{S, A, Z} c
$$

for each $c \in Z(t[\mathbf{c} / \mathbf{x}])$.

(C) If $P_{S}$ contains $t \triangleright_{S, A, Z} c$ and $t^{\prime \prime} \triangleright_{S, A, Z} c^{\prime \prime}$ such that $t^{\prime \prime}$ is a proper subterm of $t$ at $r^{\prime \prime}$, then $P_{S, A, Z}$ contains

$$
t\left[r^{\prime \prime} \leftarrow c^{\prime \prime}\right] \triangleright_{S, A, Z} c^{\prime}
$$

for each $c^{\prime} \in Z\left(t\left[r^{\prime \prime} \leftarrow c^{\prime \prime}\right]\right)$.

Define $\Rightarrow_{S, A, Z}$ as the one-step reduction relation by $\triangleright_{S, A, Z}$. Let $\Rightarrow_{S, A, Z}^{*}$ denote the reflexive and transitive closure of $\Rightarrow_{S, A, Z}$. For readability, we often write $\triangleright_{S}$ and $P_{S}$ instead of $\triangleright_{S, A, Z}$ and $P_{S, A, Z}$, respectively.

The next lemma states that each rule in $P_{I}$ is conservatively approximated by a rule in $P_{S}$.

Lemma 4..1 If there is an interpretation $I=(\nu, \mu)$ such that $t[\mathbf{o} / \mathbf{x}] \triangleright_{I} o \in P_{I}$ for some $\mathbf{0} \in \nu(\mathbf{c})$ and $o \in \nu(c)$, then $t[\mathbf{c} / \mathbf{x}] \triangleright_{S} c \in P_{S}$.

\section{Proof}

We use induction on the number of the iterations of a procedure which computes the least fixed point satisfying the three conditions in Def. 21.

Basis: Consider the case that $m(\mathbf{o}) \triangleright_{I} o(o \in \nu(c))$ is obtained from Def. 21(A). Then, $m(\mathbf{c}) \in A, \mathbf{o} \in \nu(\mathbf{c})$, and $m(\mathbf{o}) \downarrow=o$. Moreover, $c \in Z(m(\mathbf{c}))$ from the property of $Z$. From Def. 22(A), $P_{S}$ contains $m(\mathbf{c}) \triangleright_{S} c$ since $m(\mathbf{c}) \in A$ and $c \in Z(m(\mathbf{c}))$. The case that $\operatorname{Res}\left(m_{\mathrm{c}}(\mathbf{c})\right)[\mathbf{o} / \mathbf{x}] \triangleright_{I} \mathrm{o}$ is obtained from Def. $21(B)$ can be proved in the same way. 
Induction: Suppose that $t^{\prime \prime}\left[\mathbf{o}^{\prime \prime} / \mathbf{x}^{\prime \prime}\right]\left(\mathbf{o}^{\prime \prime} \in \nu\left(\mathbf{c}^{\prime \prime}\right)\right)$ is a proper subterm of $t[\mathbf{o} / \mathbf{x}]$ $(\mathbf{o} \in \nu(\mathbf{c}))$ at $r^{\prime \prime}$ and that $t[\mathbf{o} / \mathbf{x}] \triangleright_{I} o(o \in \nu(c))$ and $t^{\prime \prime}\left[\mathbf{0}^{\prime \prime} / \mathbf{x}^{\prime \prime}\right] \triangleright_{I} o^{\prime \prime}\left(o^{\prime \prime} \in \nu\left(c^{\prime \prime}\right)\right)$ have been obtained. Let $t^{\prime}\left[\mathbf{o}^{\prime} / \mathbf{x}^{\prime}\right]=t[\mathbf{o} / \mathbf{x}]\left[r^{\prime \prime} \leftarrow o^{\prime \prime}\right]\left(\mathbf{o}^{\prime} \in \nu\left(\mathbf{c}^{\prime}\right)\right)$, and suppose that $t^{\prime}\left[\mathbf{o}^{\prime} / \mathbf{x}^{\prime}\right] \triangleright_{I} o$ is obtained from Def. $21(C)$. By the inductive hypothesis, $P_{S}$ contains both $t[\mathbf{c} / \mathbf{x}] \triangleright_{S} c$ and $t^{\prime \prime}\left[\mathbf{c}^{\prime \prime} / \mathbf{x}^{\prime \prime}\right] \triangleright_{S} c^{\prime \prime}$. From the definition of $t^{\prime}\left[\mathbf{o}^{\prime} / \mathbf{x}^{\prime}\right]$, we obtain $t^{\prime}\left[\mathbf{c}^{\prime} / \mathbf{x}^{\prime}\right]=t[\mathbf{c} / \mathbf{x}]\left[r^{\prime \prime} \leftarrow c^{\prime \prime}\right]$. Since $t^{\prime}\left[\mathbf{o}^{\prime} / \mathbf{x}^{\prime}\right] \triangleright_{I} o \in P_{I}$ implies $t^{\prime}\left[\mathbf{o}^{\prime} / \mathbf{x}^{\prime}\right] \downarrow=o$, it holds that $c \in Z\left(t^{\prime}\left[\mathbf{c}^{\prime} / \mathbf{x}^{\prime}\right]\right)$. From the above inductive hypothesis and Def. 22(C), we can conclude that $t^{\prime}\left[\mathbf{c}^{\prime} / \mathbf{x}^{\prime}\right] \triangleright_{S} c \in P_{S}$.

We have the following theorem:

Theorem 2 Let $\tau \in T_{M}(C)$. If there exists no class $c$ such that $\tau \Rightarrow_{S, A, Z}^{*} c$, then $\tau$ is secure, i.e., there exists no interpretation $I=(\nu, \mu)$ such that $\tau[\mathbf{o} / \mathbf{c}] \Rightarrow_{I, A}^{*}$ ofor any $\mathbf{o} \in \nu(\mathbf{c})$ and $o \in O_{I}$.

\section{Proof}

By Lemma 4..1, it can be easily shown that if there is $I=(\nu, \mu)$ such that $t[\mathbf{o} / \mathbf{x}] \Rightarrow_{I}^{*} t^{\prime}\left[\mathbf{o}^{\prime} / \mathbf{x}^{\prime}\right]$ for some $\mathbf{o} \in \nu(\mathbf{c})$ and $\mathbf{o}^{\prime} \in \nu\left(\mathbf{c}^{\prime}\right)$, then $t[\mathbf{c} / \mathbf{x}] \Rightarrow_{S}^{*} t^{\prime}\left[\mathbf{c}^{\prime} / \mathbf{x}^{\prime}\right]$. The theorem is implied by this fact.

The proposed sufficient condition is obviously decidable, since the righthand side of each rule $\triangleright_{S, A, Z}$ is a class and therefore the "size" of the term decreases every time a rule is applied.

Example 10 We said that method uses is secure in the second case of Example 7. Actually, it is not difficult to see that $P_{S}$ has only located(Host) $\triangleright_{S}$ Room and office(Employee) $\triangleright_{S}$ Room. This implies that uses(Employee) is secure.

It is open whether the undecidability of the security problem stems only from the uncomputability of $E$. In other words, it is open whether or not the sufficient condition in Theorem 2 is also a necessary one when we use $E$ as $Z$.

\subsection{MONADIC CASE}

When a given schema is monadic, the algorithm in [10] computes $E$ in time polynomial of the size of $S$. Moreover, when a given schema is monadic and we use $E$ as $Z$, the sufficient condition in Theorem 2 is also a necessary one.

Theorem 3 Let $S$ be a monadic schema and $\tau \in T_{M}(C)$. If there exists a class $c^{\prime}$ such that $\tau \Rightarrow_{S, A, E}^{*} c^{\prime}$, then $\tau$ is insecure, i.e., there exists an interpretation $I$ such that $\tau[o / c] \Rightarrow_{I, A}^{*} o^{\prime}$ for some $o \in \nu(c)$ and $o^{\prime} \in O_{I}$. 
The proof is omitted due to the space limitation.

\subsection{COMPLEXITY}

We summarize the time complexity of deciding the sufficient condition stated in Theorem 2. Define the size of a term $t$ as $|R(t)|$, i.e., the number of occurrences of $t$. Define the description length of $\Sigma_{\mathrm{c}}$, denoted $\left\|\Sigma_{\mathrm{c}}\right\|$, as the sum of the size of all $t$ such that $(m(\mathbf{c}), t) \in \Sigma_{\mathrm{c}}$. Also, define the size of $S$, denoted $\|S\|$, as follows:

$$
\|S\|=|C|+|\leq|+|M|+\left|\Sigma_{\mathrm{b}}\right|+\left\|\Sigma_{\mathrm{c}}\right\| .
$$

Let $k$ be the maximum arity of all the methods. The height of $t$ is defined as the maximum length of the occurrences in $R(t)$. Let $L$ and $H$ be the maximum size and height of all $t$ in $\left\{t \mid(m(\mathbf{c}), t) \in \Sigma_{c}\right\} \cup\{\tau\}$, respectively. By assuming $L \leq\|S\|$, the total time complexity (including computation of $Z$ ) is

$$
\mathcal{O}\left(k^{H+1} L\|S\|^{2}(|C|+1)^{2 k^{H+1}+1} \log \|S\|\right) .
$$

Theorem 4 For a monadic method schema, the security problem is solvable in polynomial time of the size of the schema.

\section{CONCLUSIONS}

We have formalized the security problem against inference attacks on OODBs, and shown that the problem is undecidable. Then we have proposed a decidable sufficient condition for a given method to be secure, by introducing class-level inference $\left(\triangleright_{S}\right)$ which conservatively approximates object-level inference $\left(\triangleright_{I}\right)$. We believe that the approximation is fairly tight in spite of its simple definition, since the sufficient condition becomes a necessary one when the given schema is monadic.

In several situations, imprecise inference becomes powerful enough to cause serious problems. Moreover, method schemas do not seem a perfect model of OODB schemas since they do not support multi-valued methods, update operations, and so on. Therefore, we intend to extend both inference and database models.

\section{Acknowledgments}

The authors are grateful to Professor Hiroyuki Seki of Nara Institute of Science and Technology for his invaluable discussions and comments. This research is supported in part by Japan Society for the Promotion of Science under Grant-in-Aid for Encouragement of Young Scientists No. 11780306. 


\section{References}

[1] Abiteboul, S., Hull, R. and Vianu, V. (1995). Foundations of Databases. Addison-Wesley.

[2] Abiteboul, S., Kanellakis, P., Ramaswamy, S. and Waller, E. (1995). Method schemas. Journal of Computer and System Sciences, 51, pp. 433455.

[3] Bertino, E. and Samarati, P. (1994). Research issues in discretionary authorizations for object bases. Proceedings of OOPSLA-93 Conference Workshop on Security for Object-Oriented Systems, pp. 183-199.

[4] Fernandez, E.B., Larrondo-Petrie, M.M. and Gudes, E. (1993). A methodbased authorization model for object-oriented databases. Proceedings of OOPSLA-93 Conference Workshop on Security for Object-Oriented Systems, pp. 135-150.

[5] Hale, J., Threet, J. and Shenoi, S. (1994) A practical formalism for imprecise inference control. Database Security VIII: Status and Prospects, North-Holland, pp. 139-156.

[6] Hinke, T.H., Delugach, H.S. and Wolf, R. (1997). A framework for inference-directed data mining. Database Security X: Status and Prospects, Chapman \& Hall, pp. 229-239.

[7] Ishihara, Y., Shimizu, S., Seki, H. and Ito, M. (1998). The type-consistency problem for queries in object-oriented databases. Technical Report 98004, NAIST, http://isw3.aist-nara.ac.jp/IS/TechReport2/report/98004.ps.

[8] Plaisted, D.A. (1993). Equational reasoning and term rewriting systems. Handbook of Logic in Artificial Intelligence and Logic Programming, Volume 1, Oxford Science Publications, pp. 273-364.

[9] Rath, S., Jones, D., Hale, J. and Shenoi, S. (1996). A tool for inference detection and knowledge discovery in databases. Database Security IX: Status and Prospects, Chapman \& Hall, pp. 229-239.

[10] Seki, H., Ishihara, Y. and Dodo, H. (1998). Testing type consistency of method schemas. IEICE Transactions on Information and Systems, E81-D, pp. 278-287.

[11] Tajima, K. (1996). Static detection of security flaws in object-oriented databases. Proceedings of the 1996 ACM SIGMOD International Conference on Management of Data, pp. 341-352.

[12] Zhang, K. (1998). IRI: A quantitative approach to inference analysis in relational databases. Database Security XI: Status and Prospects, Chapman \& Hall, pp. 279-290. 